\title{
Observables, gauge invariance, and the role of the observers in the limit from general relativity to special relativity
}

\author{
Josep M. Pons ${ }^{a}$ \\ ${ }^{a}$ Departament d'Estructura i Constituents de la Matèria \\ Universitat de Barcelona \\ Av. Diagonal 647 \\ 08028 Barcelona \\ Catalonia, Spain \\ e-mail: pons@ecm.ub.es
}

October 30, 2018

\begin{abstract}
Some conceptual issues concerning general invariant theories, with special emphasis on general relativity, are analyzed. The common assertion that observables must be required to be gauge invariant is examined in the light of the role played by a system of observers. Some features of the reduction of the gauge group are discussed, including the fact that in the process of a partial gauge fixing the reduction at the level of the gauge group and the reduction at the level of the variational principle do not commute. Distinctions between the mathematical and the physical concept of gauge symmetry are discussed and illustrated with examples. The limit from general relativity to special relativity is considered as an example of a gauge group reduction that is allowed in some specific physical circumstances. Whether and when the Poincaré group must be considered as a residual gauge group will come out as a result of our analysis, that applies, in particular, to asymptotically flat spaces.
\end{abstract}

\section{Introduction}

Poincaré invariance and general — reparameterization, or diffeomorphism - invariance are usually considered very separate conceptual fields. Thus, whereas the former, which is a rigid symmetry, is given physical significance, the latter, which is a gauge symmetry, is considered unphysical. Gauge invariance is a symmetry of the equations of motion that depends upon arbitrary functions and their derivatives, so a given set of initial data can lead to different future evolutions that will be related by gauge transformations. A deterministic interpretation of the physical time evolution of the system then requires these gauge related trajectories to be physically equivalent. This is the case for diffeomorphism invariance in general invariant theories.

We always consider the gauge group as acting on the space of field configurations - including, if necessary, particle-like world lines. In the particular case of a general invariant theory, part of the gauge group — or the whole of it in absence of other gauge symmetries - is induced by the 
diffeomorphism group originally acting on the spacetime manifold. In this case an element of the gauge group prescribes the action of a given diffeomorphism on a given field configuration. In General Relativity (GR) this means that different metrics, and different fields in general, can undergo different diffeomorphisms [1] [2] under a single element of the gauge group. An example is, for instance, the action of the canonical generators of the gauge group in Einstein-Yang-Mills theories, where their associated transformations exhibit a compulsory dependence on the lapse and shift functions and on the time component of the gauge vector field [3] [ [4]. The gauge group in a general invariant theory is thus a diffeomorphism-induced gauge group.

Other cases of gauge invariance are associated with internal groups of symmetry, as in YangMills type theories. Instead of the ordinary conserved quantities associated - through Noether theorem - with rigid symmetries, gauge invariance leads to constraints that restrict the physical phase space. For theories exhibiting this internal gauge invariance, such as electromagnetism within the framework of Special Relativity (SR), the physical interpretation requires the observables to be gauge invariant — at least on shell- . Accordingly, in a general invariant theory, it seems natural that the observables be required to be invariant under diffeomorphisms. In contrast, no one will ask the observables in a Poincaré invariant theory to be Poincaré invariant, that is, to commute with the generators of the Poincaré algebra. There is thus a sharp contrast between the concept of observables in a general invariant theory and in SR.

General invariance is a basic principle for any physical theory because of the universality of gravitation, that couples directly to matter and energy. Wherever there is "something", there is gravity. Then the principle of equivalence, which we subscribe, will lead us to general invariance, that is, invariance under diffeomorphisms.

General Relativity (GR) is a classical example of a general invariant theory. It is common to general invariant theories to admit formulation in any set of coordinates, their physical contents being independent of the choice of the coordinates used to describe the system. But then, how can we ever use and attach physical meaning to a theory like SR, that is not general invariant? The answer is: as an approximation. The concept of a Poincaré invariant fundamental physical theory with formulation in Minkowski space is acceptable as long as the gravitational interaction is not relevant for the phenomena the theory is intended to describe. Otherwise the theory should be formulated in a general invariant way. To say it in another way: the reason why a given classical fundamental theory is not general invariant but only Poincaré invariant is because such a theory is only an approximation - that may be an extraordinarily good approximation - to a physical situation where it is reasonable to ignore gravity. Consequently, there should exist a procedure, applicable at least in some definite physical situations, to go from a general invariant theory to a Poincaré invariant one. This is the meaning for GR to be a relativistic theory [5].

Let us see how this smooth transition from general invariance to Poincaré invariance can be performed. Consider that we start with a theory of GR with matter in a physical situation such that it is allowed to neglect the gravitational interaction when compared to other, much stronger, interactions. In such cases we take the spacetime manifold as non dynamical and, moreover, we make the approximation that it is flat. What remains then of the gauge invariance associated with the freedom of reparameterization? At this point nothing seems to have changed because general invariance is still present, but the dynamics has undergone substantial changes for, being the gravitational field non dynamical, there are no longer equations of motion associated with the 
metric of spacetime. In particular, the constraints corresponding to the momenta canonically conjugate to the lapse and shift functions disappear because lapse and shift, as components of the metric, are no longer dynamical variables. They are just background. Diffeomorphism invariance is still in place in the sense that we are free to reparameterize, but there are no constraints associated with this invariance because the metric has ceased to be dynamic. At this point it may prove convenient to make a partial gauge fixing and to decide to work only with systems of coordinates such that the metric takes the Minkowski form. Notice the crucial fact that this gauge fixing is partial because some gauge transformations survive it: what is left from the diffeomorphism group after this gauge fixing is just the Poincaré group. Thus, the transition from a general invariant theory to a Poincaré invariant one satisfies an obvious consistency check, that of the reduction of the gauge group.

According to this interpretation, the Poincaré group is a leftover, that is, a residual gauge group, that appears in a general invariant theory as a byproduct of a) ignoring gravity (which must be sustained on physical grounds), and b) selecting the Minkowski form for the flat metric.

However, this construction of a smooth limit from GR to SR meets with some conceptual obstacles.

One first difficulty appears when both theories, the original general invariant theory and the reduced Poincaré invariant theory, are formulated in variational terms; in this case, the limit from GR to SR contains two different processes that do not commute: the process of reducing the gauge group, and the process of reducing the dynamics or, more properly, the process of reducing the variational principle. This issue will be explained and clarified in section 3 .

A second difficulty stems form the very concept of observables. If an observable in GR is required to be diffeomorphism invariant, our limiting procedure to SR will convert it in a Poincaré -the residual gauge group- invariant object, which is a concept very far away from the standard concept of observable in SR. So it seems as if there is a discontinuity in a limiting procedure from GR to SR, at least concerning the concept of observables. Is there any way out?

Our answer is in the positive, because so far we have been considering observables without any regard to the observers. It is time to consider them, as the piece that mediates between the observables and their physical interpretation. Let us have a look first to SR. In SR one defines privileged systems of observers: those sitting in the inertial reference frames. Once an inertial reference frame is selected, we fill it, as an idealization, of observers sitting each at any value of the space coordinates, with clocks that are synchronized. Observers in SR are then labeled (with Cartesian space coordinates, for instance) in a way that, together with their synchronized clocks, allows for a direct physical interpretation of their readings in terms of lengths and lapses of time. All these readings are values for the observables of length and time.

In contrast, observers in GR usually have a very different status, for it is not possible in general to attach direct physical significance to the coordinates they are working with. Our aim is to look for a concept of observers in GR that could eventually be consistent with the usual concept of observers in SR when the limiting procedure from GR to SR is taken.

One first approximation to observers in GR is that they do not interfere with the geometry, that is, they are idealized observers playing only a kinematical role, as test bodies. Let us examine, in the framework of GR, the basics for defining physical length and physical time, consistent with a system of observers, and keeping an eye to an eventual limiting procedure to 
SR (these considerations will be expanded with all technical details in the next section). As we have said, our observers are idealized: they must be able to gather information from their surroundings but without affecting neither each other nor the spacetime manifold. Proper time is always defined for every observer -that follows a time-like world line. Since we are free to choose coordinates, it is natural to make this choice in agreement with what we know of the observers in SR, so we choose coordinates that will be comoving with the observers, that is, the observers will sit at fixed values of the three-space coordinates. The distance from an observer to an infinitesimally close neighbor observer can be defined but it can not be extended, in general, to finite distances. In the next section we will review the conditions set for these observers in order to be consistent with a transitive synchronization that is preserved in time. In such case, we say that the observers are privileged.

These privileged observers allow for the emergence of a physical concept (always associated with this system of observers): that of equal time surfaces. We submit that this concept is "physical" - observable - not because it is diffeomorphism invariant, which is not, but because it is associated with a given system of privileged observers, in the same way as we consider physical concepts in SR (for instance: simultaneity of two events is a physical observation, though it only makes sense when associated with a given SR inertial reference system).

As long as a system of observers of this type can be set in a given general invariant theory, events will be referred to it. In this sense, standing with a given system of observers is equivalent to performing a partial gauge fixing (technical details in the next section). Now the observables need not be given in a gauge invariant (that is, diffeomorphism invariant) way but only as invariant under the residual gauge transformations that preserve the system of observers. This is the key point that will help to dissolve the conceptual difficulties we faced with the limit GR $\rightarrow$ SR. Notice also that this limit does not need to be performed in a full spacetime manifold but it can be circumscribed to a specified region of it: the spacetime region where the experiences we try to describe within our theoretical framework are taking place. On the other hand, full gauge invariance of the observables may be restored by introducing a diffeomorphism invariant description of the observers into the formalism.

The concept of observability in GR has been discussed long ago [6, 7]. It has been deeply analyzed in [B], where the introduction of the "non-local" and the "local" points of view - based upon the non-existence or existence of observers associated with a given reference system - is very illuminating. In this sense we think that our approach keeps a close relationship with, and is indebted to, [8] in that some eventual contradictions in the literature are solved by clarifying the role of observers. Our scope, though, is somewhat different, for we try to meet the conditions to produce a smooth transition from a general invariant theory to a Poncaré invariant theory.

The paper is organized as follows. In the next section we technically substantiate the discussion introduced above. In section 3 we make some distinctions between the physical and the mathematical concept of gauge transformations, and a subsection is devoted to a specific example featuring some of these ideas. Further applications of these ideas to asymptotically flat spacetimes are given in section 4 . Finally section 5 is devoted to conclusions. 


\section{Privileged systems of observers in GR}

In GR, or in any general invariant theory, given (at least in the spacetime region of our interest) a three-parametric congruence of time-like world lines - that is, a platform [9]-, we can always take coordinates in a $3+1$ decomposition adapted to this congruence, such that each world line has a fixed value for the space three-coordinates (comoving coordinates). We can think of observers attached to these world lines. Then, by use of light rays one can define a synchronization between one observer and another infinitesimally close. The vector fields that connect synchronized infinitesimally close neighbor observers are

$$
\mathbf{X}_{i}:=\partial_{i}-\frac{g_{0 i}}{g_{00}} \partial_{0}
$$

where $g_{\mu \nu}(\mu, \nu=0, \ldots, 3 ; i=1,2,3)$ are the components of the spacetime Lorentzian metric. The vectors $\mathbf{X}_{i}$ span the directions orthogonal to the vector field tangent to the world lines.

A good synchronization is characterized by two properties: first, it must be consistent with that of the neighbors' (transitive property for infinitesimally close observers: if $A$ is synchronized with $B$, and $B$ with $C$, then $A$ and $C$ must be synchronized); and second, it must be kept in time (to be a physical synchronization: If $A$ and $B$ are synchronized at time zero, they must be so at later times).

Transitivity of the synchronization. The first property is equivalent to saying that the vector fields $\mathbf{X}_{i}$ form a distribution, that is, they locally define a three-surface. Due to the particular form of $\mathbf{X}_{i}$, this property amounts to commutativity:

$$
\left[\mathbf{X}_{i}, \mathbf{X}_{j}\right]=0 \text {. }
$$

This property is equivalent to that of the irrotationallity of the world lines of our observers. A weaker sense of consistency can also be defined for a two-parametric family of observers. For instance, the property

$$
\left[\mathbf{X}_{1}, \mathbf{X}_{2}\right]=0
$$

already guarantees the existence of two-surfaces such that observers sitting in those surfaces (a two-parametric family of observers) can be consistently (transitive property) synchronized.

Preservation of the synchronization. Now enters the second property for a good synchronization: preservation in time. Neighbor observers sitting in $x^{i}$ and $x^{i}+\epsilon^{i}$, with $\epsilon^{i}$ some infinitesimal constants, will preserve their synchronization (initially produced with the help of the vector field $\left.\epsilon^{i} \mathbf{X}_{i}\right)$ ) along the time evolution if and only if

$$
\left[\epsilon^{i} \mathbf{X}_{i}, \mathbf{X}_{0}\right]=0
$$

where $\mathbf{X}_{0}$ is the vector field generating the evolution in proper time:

$$
\mathbf{X}_{0}:=\frac{1}{\sqrt{-g_{00}}} \partial_{0} .
$$

The interpretation of (3) is obvious: displacement to the neighbor observer and evolution in proper-time commute. If the preservation in time of the synchronization is required for any set of $\epsilon^{i}$, then the condition is:

$$
\left[\mathbf{X}_{i}, \mathbf{X}_{0}\right]=0
$$


Equations (11) and (4) describe the full compatibility of a system of observers with a synchronization that is preserved in time.

Geodesic condition. Up to now we have said nothing regarding the motion of our observers in spacetime. In this respect, we must take notice that equations (41) are exactly the conditions for our observers to follow a geodesic of the metric [10]. The motion of the privileged observers is free fall.

In conclusion, irrotationallity (1) and geodicity (任) are the two conditions that our world lines must satisfy in order to be associated with privileged observers in GR. This corresponds to a platform that is locally proper-time Einstein-synchronizable [9, 11].

Equation (1) implies that we can make a change of coordinates

$$
\left(x^{i}, x^{0}\right) \Rightarrow\left(x^{i}, x^{\prime 0}\right)
$$

such that $\mathbf{X}_{i}$ becomes $\partial_{i}^{\prime}$ in the new coordinate system . This leads to $x^{\prime i}=x^{i}$ (the observers still sit in the same values of the three-coordinates) and to a function $x^{\prime 0}\left(x^{i}, x^{0}\right)$, solution of the equations

$$
\mathbf{X}_{i}\left(x^{\prime 0}\right)=0 .
$$

The metric tensor is rewritten, under this change of coordinates, as

$$
g_{0 j}^{\prime}=0, \quad g_{i j}^{\prime}=g_{i j}-\frac{g_{i 0} g_{0 j}}{g_{00}}, \quad g_{00}^{\prime}=g_{00} \frac{1}{\left(\frac{\partial x^{\prime 0}}{\partial x^{0}}\right)^{2}} .
$$

The interpretation of (7) is clear. According to the first equation, the lapse functions vanish in the new coordinate system. As regards the second equation, $g_{i j}-\frac{g_{i 0} g_{0 j}}{g_{00}}$ is just the Landau metric [12] that defines the spatial distance between infinitesimally close observers in comoving coordinates; since in the new coordinates these observers are synchronized, their spatial distances are directly determined by the new 3 -metric $g_{i j}^{\prime}$. The last equation is a consistency requirement for the invariance of proper times as computed in the old or in the new system of coordinates.

In the new system of coordinates, the condition (何) for the preservation of the synchronization has a new interpretation: $g_{00}^{\prime}$, when expressed in the new coordinates, must be a function of $x^{\prime 0}$ exclusively. Then the proper time between $x_{A}^{\prime 0}$ and $x_{B}^{\prime 0}$ is independent of the observer, that is, it will not depend on the three-coordinates, which means that synchronization will be preserved in time. It is easy to verify that the requirement

$$
\partial_{i}^{\prime}\left(g_{00}^{\prime}\right)=0
$$

is equivalent to (4), provided, of course, the fulfillment of (1).

With the new coordinates the metric takes the form (we drop the primes)

$$
\left(g_{\mu \nu}\right)=\left(\begin{array}{cc}
g_{00}\left(x^{0}\right) & 0 \\
0 & g_{i j}\left(x^{0}, x^{i}\right)
\end{array}\right)
$$

and now, with a simple reparameterization of the time coordinate, we can get $g_{00}=-1$, which means that we are using the proper time of the synchronized observers as the new time coordinate. 
Obviously, a system of observers in free fall is not always physically realizable. It is common in cosmology, when the observers can be idealized as comoving with the galaxies (up to the peculiar motions), but if we study the motion of a particle around a Schwarzchild solution of Einstein equations, it is not the kind of observers we will use. Notice, however, that in this last example gravity can not be neglected, and we are interested in cases where a smooth transition from GR to SR can be taken.

Coordinates such that the metric satisfies

$$
g_{00}=-1, \quad g_{0 j}=0
$$

are known as Gaussian coordinates [14, 15, 10]. Obviously a suitable gauge transformation - a diffeomorphism - can always set the coordinates in a local patch to be Gaussian. Gaussian coordinates are associated therefore to inertial (that is, moving along geodesics) comoving observers, that are synchronized to each other and such that their clocks give the time coordinate (proper time) for an event. These families of observers associated with Gaussian coordinates (or coordinates that can be put in Gaussian form by a change of the type (5)) are therefore privileged families of observers. This is as close as we can get in GR to the usual systems of observers of SR.

Infinitesimal coordinate transformations $x^{\mu} \rightarrow x^{\mu}-\epsilon^{\mu}$ that keep $g_{00}=1$ and $g_{0 j}=0$ must satisfy (the comma denotes partial differentiation):

$$
\epsilon_{, 0}^{0}=0, \quad \epsilon_{, i}^{0}=g_{i j} \epsilon_{, 0}^{j} .
$$

Notice that (9) implies that $\vec{\epsilon}_{, 0}=0$ if and only if $\nabla \epsilon^{0}=0$, where $\vec{\epsilon}=\left(\epsilon^{1}, \epsilon^{2}, \epsilon^{3}\right)$ and $\nabla \epsilon^{0}=\left(\partial_{1} \epsilon^{0}, \partial_{2} \epsilon^{0}, \partial_{3} \epsilon^{0}\right)$. So when, in addition to (9), we have $\nabla \epsilon^{0}=0$, the transformation amounts to a relabeling of the spatial coordinates for the observers and a rigid translation of the zero of time: the system of observers remains the same. Instead, when $\nabla \epsilon^{0} \neq 0$, the change of spatial coordinates involves the time and therefore the system of observers changes (these are the "boosts" in GR). In order to do physics it is legitimate to stay with a given system of observers and to make a choice of the zero of time for their synchronized clocks. These choices amount to a partial gauge fixing. Under this interpretation, physics does not require our observables to be invariant under general diffeomorphisms, but only under diffeomorphisms that are compatible with the system of observers we are working with - but see the remarks below. In this sense, a distance as given by the three-metric is an observable (because it is invariant under the arbitrary three-space diffeomorphisms described by (9) when restricted to $\nabla \epsilon^{0}=0$ ). This distance may change in time if $g_{i j}$ has a time dependence; this only means that such distance, always referred to our system of observers, is not time invariant, but it is still physical, observable.

This discussion suggests that the usual claim that, in general invariant theories, only gauge invariant quantities, that is, diffeomorphism invariant quantities, are acceptable as observables, must be re-analyzed. Diffeomorphism invariance must be required if we lack of a system of observers of the type we have been building up, but not when it is possible to set up a system of observers consistent with synchronization. Then we only need to ask the observables to be invariant under transformations that do not change the family of observers. Once said that, let 
us notice that the alternative, fully diffeomorphism invariant description, can always be restored by including the observer's world-lines in the physical description. See the remarks below.

Summing up, we have cut the original set of gauge transformations along several steps. In the first step, only gauge transformations preserving the Gaussian condition are allowed. In the second, the gauge group is further restricted to the transformations that preserve the system of observers (three-diffeomorphisms and proper time translations), and finally, by deciding the zero of the time coordinate, we are left with the three-diffeomorphisms as the residual gauge freedom. The transformations that change the system of observers are interpreted as a change of description, from one system of observers to another. These transformations have some analogy with the boosts in SR.

Now consider that, in a given region of spacetime, a smooth transition to SR can be taken. Recall that with our privileged observers in GR we have already fixed the gauge freedom of time translations by deciding the zero of the time coordinate, now in SR we can even decide where to place the origin (the zero) of the three-coordinates and to choose three orthogonal space directions to be labeled as the $x, y, z$ directions. All these choices are gauge fixings, and we can then agree that our descriptions of physical events will be referred to this fixed framework. This means that we have finally shrunk the residual gauge group to nothing and, therefore, there are no longer gauge invariance requirements for our observables. The result is that a simple position at a given time, the third component of an angular momentum, etc., become observables. Thus observability increases a great deal when the limit is taken from GR to SR (by neglecting gravity when it is physically acceptable), for Cartesian coordinates in the latter are endowed with a direct physical significance that has no parallel in the former. If in our previous discussion within GR we found that the relational concept of observables had room for systems of observers that will refer the physical events in a three-space diffeomorphism invariant way, now in SR the system of observers, directly associated with a given Cartesian coordinate frame, carries direct physical observable content, thus enlarging enormously the concept of observability. This explains why the concepts of observability look so different when considered in GR or in SR.

Let us finally make three important remarks.

First, our observers can be conceived as test bodies [13] forming a grid of sensors moving in free fall that, in an acceptable approximation for the phenomena they are set to describe, collect information from the media without producing or receiving any other (significant) perturbation in or from it . Observers, thus, must be physical. Whether they account or not for some matter degrees of freedom depends on the approach we take for their description, as is clarified in the next remark. They are part of the -classical- physical system but with an ideal beauvoir -a non-interference condition- with respect to the rest of it. In this case, the relational concept 16] of observables in GR, which is the statement that objects are only localized with respect to other objects and not with respect to background space, can take advantage of this structure of observers added to the physical framework, and become a concept suited to perform the limit $\mathrm{GR} \rightarrow \mathrm{SR}$.

Second. According to the precedent discussions, the observables prepared for a smooth limit from GR to SR admit two interpretations; one coming from a gauge fixing procedure, the other

\footnotetext{
${ }^{1}$ These idealized conditions are close to those introduced in section 3 of 8 for the material reference systems.
} 
keeping the full gauge freedom. In the gauge fixing approach, which is the one we have been introducing, we select a gauge such that the coordinates are Gaussian and comoving with a given system of observers; this choice of coordinates is the only effect of the presence of the observers in our physical framework. Now the observables must only be gauge invariant in a restricted sense (under the gauge transformations that preserve the system of observers). If we wish to restore the full diffeomorphism invariance of the observables we must first go back to a diffeomorphism invariant description of the observers. This is the second interpretation of the observables: as long as observers enter the stage, the search for observables becomes the search for diffeomorphism invariant quantities made up with the dynamical entities (particles and fields) actively present in the physical scenario plus the observers' world lines, that are kinematical entities playing a passive role in the physical setting.

Third. In many physical applications of GR the system of observers is not of the type discussed here. For instance, in terrestrial laboratory experiments of the gravitational redshift, observers sitting in different radial coordinates can not be synchronized. In fact, this impossibility of synchronization is deeply related to the gravitational redshift. On the other hand, in experiences of observation of the bending of light in a path from the stars passing close to the Sun, observers are considered as placed in an asymptotic region and so they satisfy our conditions. In conclusion, there are different ways to place observers in a region of spacetime and not all of them are suited for a limiting procedure to SR.

\section{Mathematical versus physical gauge freedom. Some gauge fixing surprises.}

Consider theories derived from a variational principle. From the purely mathematical perspective, infinitesimal symmetries whose parameters are arbitrary functions are gauge, whereas infinitesimal symmetries whose parameters are (infinitesimal) constants are rigid. This is mathematics. Gauge transformations will be associated with and generated by constraints in the Dirac sense, whereas rigid symmetries will be associated with and generated by ordinary constants of motion. From the point of view [17] of physical determinism, mathematical gauge symmetries must correspond to transformations that do not change the physical state, the reason being that gauge transformations can link different solutions of the equations of motion that satisfy the same set of initial conditions and therefore these different solutions must be considered physically equivalent.

So far the mathematical and physical concepts of gauge and rigid symmetries are in quite a trivial correspondence. But exceptions abound. The first exception has been already commented upon in the introduction: in the process of a partial gauge fixing the reduction at the level of the gauge group and the reduction at the level of the variational principle do not necessarily commute. Let us clarify this important point by considering again the limiting procedure. 


\subsection{Problems associated with a partial gauge fixing}

We start with a full-fledged general invariant theory described by a Lagrangian density

$$
\mathcal{L}\left(g_{\mu \nu}, \phi^{a}, g_{\mu \nu, \sigma}, \phi_{, \sigma}^{a}, \ldots\right)
$$

where $\phi^{a}$ represent the fields other than the metric (there can be boundary terms for the action, in order to guarantee that the variational principle yields the right equations of motion). $\mathcal{L}$ is supposed to be a scalar density. Gauge freedom for this theory will contain the diffeomorphism invariance and perhaps other invariances (for instance, in an Einstein-Yang-Mills theory there will be the gauge invariance associated with the YM part).

Next, consider the limiting case where gravitational effects can be neglected and then choose a gauge fixing that sets $g_{\mu \nu}=\eta_{\mu \nu}$ everywhere (or just in the region of spacetime we are interested

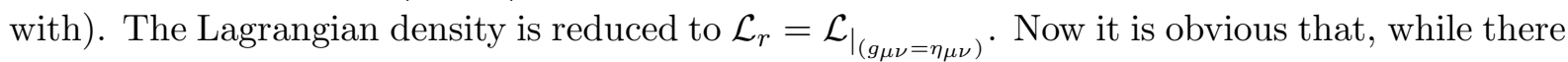
remains the residual gauge freedom associated with the diffeomorphisms that keep invariant the form of the metric $\eta_{\mu \nu}$ (that is: the Poincare transformations), this residual gauge freedom can no longer be retrieved from $\mathcal{L}_{r}$ as such gauge freedom in the mathematical sense, for the only mathematical gauge freedom available in $\mathcal{L}_{r}$, if any, comes from the other invariances that could exist in the original Lagrangian besides diffeomorphism invariance.

Therefore, when one is presented with a Lagrangian like $\mathcal{L}_{r}$ in the framework of SR, there are two ways for its physical interpretation: either a) we conceive it as the - classical - ultimate description of the physical system we are dealing with, and in such case the gauge freedom will only be the one mathematically derived from $\mathcal{L}_{r}$; or b) we consider that any fundamental Lagrangian that neglects gravity is only an approximation to the correct theory; this correct theory implementing general invariance (There are methods to reintroduce a dynamical metric -or a tetrad- within $\mathcal{L}_{r}$ in order to make it general invariant). In this case, the physical gauge freedom available for $\mathcal{L}_{r}$ may be larger that the mathematically derived from it. If we think a), then Poincaré group is not gauge. If we think b), Poincaré group is the residual gauge group that is left after the introduction of the gauge fixing $g_{\mu \nu}=\eta_{\mu \nu}$. As long as Poincaré invariant theories are conceived as approximations where the role of gravity has been deemed irrelevant, the correct interpretation is b), but the second remark at the end of section 2 shows that the final answer depends on the way we deal with observers and observables. The two choices, a) and b), are available, and indeed the choice a) is the most usual in the framework of SR.

We refer to [18] for an example where a reduction process similar to the one presented above is fully accomplished in Bianchi-type cosmologies. In that case, a partial gauge fixing and the implementation of the Killing symmetries leads to a reduced Lagrangian whose mathematically associated gauge group is the group of time reparameterizations; instead, from the point of view of reducing the gauge group, in addition to time reparameterizations there remain, as part of the residual gauge group, some special three-space diffeomorphisms - the homogeneity preserving diffeomorphisms [20]. Therefore, we are unable to retrieve the full residual gauge group from the information provided by the reduced Lagrangian itself. The gauge group mathematically associated with the reduced Lagrangian is a subgroup of the complete residual gauge group. The physical consequences of this analysis are immediate, because the number of physical degrees of freedom depends upon the interpretation we take. 
It is worth mentioning that the reduced Lagrangian might suffer other drawbacks: constraints may be lost in the process of reduction of the variational principle (see [19] [18]), even though the variables involved in these constraints are not eliminated in the reduction process. The only remedy for this problem is to reintroduce from the outset these lost constraints as restrictions on the initial conditions.

\subsection{Problems associated with boundary conditions}

Another source of conflict between the mathematical and the physical concept of gauge symmetry comes from boundary considerations. Here we examine a toy model where the "mathematical" and the "physical" concepts of gauge symmetry do not coincide because of this effect. This example is inspired in an earlier model introduced in [21].

Consider the following action in $1+1$ dimensions with fields $K, M, N$

$$
S=\int_{t_{1}}^{t_{2}} d x L, \quad L=\int_{x_{1}}^{x_{2}} d x \mathcal{L}=\int_{x_{1}}^{x_{2}} d x(K(x, t) \dot{M}(x, t)-N(x, t) M(x, t))
$$

This model exhibits the gauge symmetry

$$
\begin{aligned}
\delta K & =-\epsilon(x, t) \\
\delta M & =0 \\
\delta N & =\dot{\epsilon}(x, t),
\end{aligned}
$$

with $\epsilon$ arbitrary. Under this transformation,

$$
\delta \mathcal{L}=\frac{d}{d t}(-\epsilon M)
$$

Although the model lacks physical interpretation, we will accept (10) as the "physical" gauge freedom. In principle, regarding the boundary conditions for the application of the variational principle, one could consider that the gauge transformations are restricted to satisfy $\epsilon\left(x, t_{1}\right)=$ $\epsilon\left(x, t_{2}\right)=0$. But consider the piece of trajectory between $t_{1}^{\prime}$ and $t_{2}^{\prime}$, for $t_{1}<t_{1}^{\prime}<t_{2}^{\prime}<t_{2}$. Now $\epsilon\left(x, t_{1}^{\prime}\right)$ and $\epsilon\left(x, t_{2}^{\prime}\right)$ are free, and yet the transformed trajectory must be gauge equivalent to the original trajectory. So we conclude that the variational principle by itself imposes no restrictions to the boundary values of the arbitrary function $\epsilon(x, t)$.

Let us now introduce a boundary condition on the solutions of the equations of motion. We choose, inspired in [21],

$$
\int_{x_{1}}^{x_{2}} d x N(x, t)=\text { constant }
$$

This boundary condition restricts our gauge freedom, for we need

$$
0=\int_{x_{1}}^{x_{2}} d x \delta N(x, t)=\int_{x_{1}}^{x_{2}} d x \dot{\epsilon}(x, t) .
$$

Therefore our physical gauge freedom is now (10) supplemented with

$$
\int_{x_{1}}^{x_{2}} d x \dot{\epsilon}(x, t)=0
$$


or, equivalently,

$$
\int_{x_{1}}^{x_{2}} d x \epsilon(x, t)=\text { constant }
$$

Let us now proceed to the canonical analysis of the model.

The momenta, as functions in tangent space, are

$$
\begin{aligned}
\hat{P}_{K} & =\frac{\partial L}{\partial \dot{K}}=0 \\
\hat{P}_{M} & =\frac{\partial L}{\partial \dot{M}}=K \\
\hat{P}_{N} & =\frac{\partial L}{\partial \dot{N}}=0
\end{aligned}
$$

and the Dirac Hamiltonian becomes

$$
H_{D}=\int_{x_{1}}^{x_{2}} d x\left(N M+\lambda P_{K}+\mu\left(P_{M}-K\right)+\eta P_{N},\right.
$$

with $\lambda, \mu, \eta$ arbitrary functions.

The constraints $P_{K}$ and $P_{M}-K$ are second class and can be eliminated with the introduction of the Dirac bracket. $K$ becomes the canonical conjugate to $M$, with the equal-time bracket

$$
\{M(x, t), K(y, t)\}=\delta(x-y) .
$$

The bracket $\left\{N(x, t), P_{N}(y, t)\right\}$ needs more work, because of the boundary condition (11). Expansion in Fourier modes for the space variable tells us that all the modes for $N(x, t)$ are configuration variables except the zero mode, which is a constant according to (11). The canonical conjugates of the non-zero modes define the canonical conjugate variables. Therefore the spatial Fourier expansion of $P_{N}(y, t)$ gives a similar picture: the zero mode is absent and the non-zero modes are the variables. Should all the modes be variables, the completion formula would give $\left\{N(x, t), P_{N}(y, t)\right\}=\delta(x-y)$, but we must subtract to it the contribution of the zero modes. We find

$$
\left\{N(x, t), P_{N}(y, t)\right\}=\delta(x-y)-\frac{1}{x_{2}-x_{1}},
$$

which is consistent with

$$
\left\{\int_{x_{1}}^{x_{2}} d x N(x, t), P_{N}(y, t)\right\}=\left\{N(x, t), \int_{x_{1}}^{x_{2}} d y P_{N}(y, t)\right\}=0
$$

Let us look for the secondary constraints, Under our Dirac bracket, the Dirac Hamiltonian is just $H_{D}=\int_{x_{1}}^{x_{2}} d x\left(N M+\eta P_{N}\right)$. Stabilization of the constraint $P_{N}=0$ gives

$$
0=\dot{P}_{N}(x, t)=\left\{P_{N}(x, t), H_{D}\right\}=-M(x, t)+\frac{1}{x_{2}-x_{1}} \int_{x_{1}}^{x_{2}} d y M(y, t)=:-\Psi(x, t)
$$

This constraint $\xi(x, t)=0$ is equivalent to the equation of motion $M^{\prime}(x, t)=0$ ( $\left(M^{\prime}\right.$ stands for the space derivative of $M)$. This new constraint is first class, and there are no more constraints.

\footnotetext{
${ }^{2} M^{\prime}(x, t)=0$ is a Lagrangian equation of motion because the variation of the Lagrangian with respect to the variable $N(x, t)$ must take into account the boundary condition (11). The Lagrangian is only varied with respect to the non-zero modes of $N(x, t)$ and the associated equation of motion imposes that the non-zero modes of $M(x, t)$ vanish. This is the contents of $M^{\prime}(x, t)=0$.
} 
Let us construct the Noether gauge generator [22, 23] provided by the first class constraints. The mathematical construction gives

$$
G_{m a t h}=\int_{x_{1}}^{x_{2}} d x\left(\epsilon(x, t) \Psi(x, t)+\dot{\epsilon}(x, t) P_{N}(x, t)\right)
$$

which is subject to the restriction that it must generate transformations that respect (11). This is the case indeed because, with the Dirac brackets determined before, we find

$$
\begin{aligned}
\delta K & =\left\{K, G_{\text {math }}\right\}=-\bar{\epsilon}(x, t) \\
\delta M & =\left\{M, G_{\text {math }}\right\}=0 \\
\delta N & =\left\{N, G_{\text {math }}\right\}=\dot{\bar{\epsilon}}(x, t),
\end{aligned}
$$

(to be compared with (10) and (12)) where we have defined

$$
\bar{\epsilon}(x, t)=\epsilon(x, t)-\frac{1}{x_{2}-x_{1}} \int_{x_{1}}^{x_{2}} d y \epsilon(y, t) .
$$

Notice that $\bar{\epsilon}$ satisfies

$$
\int_{x_{1}}^{x_{2}} d y \bar{\epsilon}(y, t)=0
$$

which is in fact more restrictive than (12). The most general function $\epsilon(x, t)$ satisfying (12) is

$$
\epsilon(x, t)=\bar{\epsilon}(x, t)+a,
$$

with $\bar{\epsilon}(x, t)$ satisfying (19) and $a$ an arbitrary constant

(Note that $a$ is retrieved from $\epsilon(x, t)$ satisfying (12) as

$$
a=\frac{1}{x_{2}-x_{1}} \int_{x_{1}}^{x_{2}} d x \epsilon(x, t)
$$

$G_{m a t h}$ is equivalently written as

$$
G_{m a t h}[\bar{\epsilon}]=\int_{x_{1}}^{x_{2}} d x\left(\bar{\epsilon} M+\dot{\bar{\epsilon}} P_{N}\right)
$$

(Equation (19) makes $G_{\text {math }}[\bar{\epsilon}]$ to vanish on-shell) Notice that the complete gauge generator of the symmetry (10), with the restriction (12) is not described by (21). Indeed the physical gauge generator is

$$
G_{p h y s}[\epsilon]=\int_{x_{1}}^{x_{2}} d x\left(\epsilon M+\dot{\epsilon} P_{N}\right),
$$

with $\epsilon$ satisfying (12). Using the decomposition (20) we find

$$
G_{\text {phys }}[\epsilon]=G_{m a t h}[\bar{\epsilon}]+\int_{x_{1}}^{x_{2}} d x a M(x, t) .
$$

The first term in (23), $G[\bar{\epsilon}]$, is made up with first class constraints, because (17) and (21) are equivalent when $\epsilon$ satisfies (12); the second term, $\int_{x_{1}}^{x_{2}} d x a M(x, t)$, is made up with the constant of motion $M(x, t)$. So we see that in this model the gauge symmetry (in the physical sense) is generated both by constraints and by constants of motion. 
Now we can proceed to a gauge fixing. We fix $N(x, t)$ to be a given configuration $N_{0}(x, t)$ satisfying (11). The reduced Lagrangian becomes

$$
\mathcal{L}_{r}=K \dot{M}-N_{0} M .
$$

The residual gauge freedom is generated by the second term in (23). It gives the transformations

$$
\delta K=-a, \quad \delta M=0,
$$

which is a -physical- gauge transformation generated by a constant of motion. Notice, though, that there is no -mathematical- gauge freedom stemming directly from $\mathcal{L}_{r}$ because its two canonical constraints are second class.

\section{Asymptotically flat spaces: Poincaré group as gauge group}

The study of asymptotically flat spaces in GR has led to a distinction between what is physically gauge and what is not that has become commonplace [24]. Briefly, diffeomorphisms that become the identity at space infinity are taken as gauge, while diffeomorphisms that "move the space boundary" are considered to have a non gauge part that can be identified with the Poincaré group. Let us be more explicit.

Here we follow Faddeev approach [24]. Asymptotically flat spaces correspond to physical situations where the gravitating masses and matter fields at finite times are effectively concentrated in a finite region of space. In a topologically simple spacetime manifold whose points can be uniquely parametrized by four coordinates $x^{\mu},-\infty<x^{\mu}<\infty$, a system of coordinates admissible in order to define an asymptotically flat space is such that, in the limit $r \rightarrow \infty$ $\left(r^{2}=\left(x^{1}\right)^{2}+\left(x^{2}\right)^{2}+\left(x^{3}\right)^{2}\right)$ for finite time $t=x^{0}$, the metric components satisfy the asymptotic conditions

$$
g_{\mu \nu}=\eta_{\mu \nu}+O\left(\frac{1}{r}\right), \quad \partial_{\sigma} g_{\mu \nu}=O\left(\frac{1}{r^{2}}\right), \quad \boldsymbol{\Gamma}_{\mu \nu}^{\sigma}=O\left(\frac{1}{r^{2}}\right) .
$$

A first partial gauge fixing is clearly under way: we will only accept changes of coordinates that preserve (24). For an infinitesimal change $x^{\mu} \rightarrow x^{\prime \mu}=x^{\mu}-\epsilon^{\mu}(x)$, this means that $\epsilon^{\mu}$ must asymptotically behave as

$$
\begin{aligned}
\epsilon^{\mu} & =\omega_{\nu}^{\mu} x^{\nu}+a^{\mu}+O\left(\frac{1}{r}\right), \\
\partial_{\nu} \epsilon^{\mu} & =\omega_{\nu}^{\mu}+O\left(\frac{1}{r^{2}}\right) \\
\partial_{\nu} \partial_{\sigma} \epsilon^{\mu} & =O\left(\frac{1}{r^{2+\alpha}}\right), \alpha>0 .
\end{aligned}
$$

$\left(\omega_{\nu}^{\mu}\right.$ is an infinitesimal Lorentz parameter, $\eta_{\rho \mu} \omega_{\nu}^{\mu}+\eta_{\nu \mu} \omega_{\rho}^{\mu}=0$, and $a^{\mu}$ is an infinitesimal translation of the coordinates) Under this partial gauge fixing, our remaining diffeomorphisms group $G$ is the one generated by the transformations satisfying (25). This group has a normal subgroup $G_{0}$ generated by the transformations that become the identity for $r \rightarrow \infty$. The factor group is just the Poincaré group. It is then usual to take the normal subgroup as the physical gauge group of the theory whereas the Poincaré group, is considered as non-gauge. 
Intuitively, this procedure of singling out the Poincaré group as non-gauge relies on the consideration that the Poincaré group in SR is never taken as a gauge group, and since in our case, when $r$ goes to infinity our spacetime manifold becomes indistinguishable from Minkowski space, this Poincaré group that "moves the boundary" should not be taken as gauge. On the other hand, the Hamiltonian density for this theory [25] [24] differs from the Hamiltonian constraint by a space divergence, and the computation of the energy becomes an integration over a closed two-dimensional surface $S$ in the limit $r \rightarrow \infty$ :

$$
E=\lim _{r \rightarrow \infty} \int_{S} d \sigma_{i}\left(\partial_{j} g_{i j}-\partial_{i} g_{j j}\right) .
$$

Then, keeping the interpretation of Poincaré group as non-gauge, $E$ is invariant under the "gauge transformations" generated by $G_{0}$. Instead, since a Lorentz transformation will change the value of $E$, the energy would not be a gauge invariant concept should Poincaré group be taken as gauge.

Now our considerations on observers in GR and SR made in sections 1 and 2 enter the stage: as long as a system of observers is not introduced, the Poincaré group must be taken as part of the gauge group. According to this view, the concept of energy is no longer gauge invariant. The rationale of this conclusion can be made even more clear with the simplest of the examples. Consider in Minkowski spacetime a physical system consisting of a single particle. If, strictly, this is the whole physical system, there is no room for observers, nor even in SR. We can describe the particle motion in a given Cartesian coordinate frame, but we can not attach a physical sense -as a reference frame related to a system of observers- to that particular description. We are bound to recognize that the energy, or the momentum, of the particle, are no longer gauge invariant concepts, for there are no observers to which these concepts can refer. Any Minkowski spacetime with a single particle (the same type of particle), whatever the coordinate description we take for its motion, is always the same physical situation. The perhaps surprising fact, then, that constants of motion - not constraints - may generate physical gauge transformations though, mathematically, they are rigid transformations - is less surprising after the discussion of the toy example of subsection 3.2.

We conclude that the common assertion that diffeomorphisms that change the space boundary are not gauge transformations (that is, that they change the physics) is, face value, too rash an assertion, and that it can only make sense after the role of the observers is considered.

\section{5 conclusions}

We have discussed the role of observers in GR in view of the limiting procedure from GR to SR when gravitational effects can be ignored. We define a system of privileged observers in GR that keep good properties of synchronization with their neighbors. The world lines of these systems of observers are geodesics and irrotational. This system of observers is ideally constituted by test bodies that gather information from their surroundings but do not affect neither each other nor the spacetime manifold. When the the limit is taken from GR to SR they will correspond to the standard concept of observers used in SR. 
We show that, with regard to a physical description through a system of observers, two interpretations can be given. In the first, a partial gauge fixing is made in order to keep the coordinates comoving with the observers; then the observables must only be invariant under the elements of the diffeomorphism group that preserve the system of observers. This is not in contradiction with the relational concept of observables [8] because the readings of the observables are referred to the system of observers. In fact, this consideration leads to the second interpretation: when the world lines of the observers are included in the physical scenario, the same observables can be described as gauge invariant quantities.

Our claim is that spacetime gauge symmetry - diffeomorphism invariance - is special among all gauge symmetries, and what makes it special is the role of the observers.

We also discuss several issues that suggest the relevance of distinguishing, in some situations, between a physical concept of gauge transformation and a mathematical one, because they do not always coincide. Problems that raise this issue include reduction procedures, where we show that the reduction at the level of the gauge group and the reduction at the level of the variational principle do not necessarily commute, and other problems originated by the setting of boundary

conditions. In particular, we discuss the gauge group for asymptotically flat spaces, and we conclude in this case that the Poincaré transformations, that change the space boundaries, must be interpreted as gauge transformations unless we have placed a system of observers on which our previous discussion applies.

\section{References}

[1] J. M. Pons, D. C. Salisbury and L. C. Shepley, Phys. Rev. D55, 658 (1997) gr-qc/9612037.

[2] J. M. Pons, D. C. Salisbury and L. C. Shepley, gr-qc/9912086. J. Math. Phys....

[3] D. C. Salisbury and K. Sundermeyer, Phys. Rev. D 27, 740-756 (1983)

[4] D. C. Salisbury and K. Sundermeyer, Phys. Rev. D 27, 757-763 (1983)

[5] C. M. Will in "General Relativity, an Einstein centenary survey", S. W. Hawking and W. Israel ed. Cambridge University Press 1979.

[6] P.G. Bergmann, Reviews of Modern Physics 33, 510-514 (1961).

[7] P.G. Bergmann, "The General Theory of Relativity" in Handbuch der Physik (S. Fluegge, Ed.), Vol. IV, p. 203, Springer-Verlag, Berlin and Heidelberg, 1962.

[8] C. Rovelli, Class. Quant. Grav. 8, 297 (1991).

[9] M.H. Soffel, "Relativity in Astrometry, Celestial Mechanics and Geodesy", Springer, Berlin 1989.

[10] R. M. Wald, General Relativity (University of Chicago Press, Chicago, 1984) 
[11] R. K. Sachs and H. Wu, "General Relativity for Mathematicians", SSSpringer, Berlin, Heidelberg, News York, 1977.

[12] L. D. Landau and E. M. Lifshitz, "The Classical theory of fields", 4th edition, Oxford. Pergamon Press 1983.

[13] Y. Vladimirov, N. Mitskievich and J. Horsky, "Space, Time,Gravitation", Mir publishers, Moscow 1987.

[14] H. Weyl, "space, time, matter", 4th edition, Dover 1952.

[15] H. Ohanian and R. Ruffini, "Gravitation and space-time." New York, USA: Norton (1994) $679 \mathrm{p}$.

[16] C. Rovelli, Plenary lecture given at 15th International Conference on General Relativity and Gravitation (GR15), Pune, India, 16-21 Dec 1997. gr-qc/9803024.

[17] P. A. M. Dirac, "Lectures on Quantum Mechanics". Belfer Graduate School of Science, Yeshiva University, New York 1964.

[18] J. M. Pons and L. C. Shepley, Phys. Rev. D58, 024001 (1998) gr-qc/9805030.

[19] J. M. Pons, Int. J. Mod. Phys. A11, 975 (1996) hep-th/9510044.

[20] A. Ashtekar and J. Samuel, Class. Quant. Grav. 8, 2191 (1991)

[21] D. Marolf, Class. Quant. Grav. 13, 1871 (1996) gr-qc/9510023.

[22] C. Batlle, J. Gomis, X. Gracia and J. M. Pons, J. Math. Phys. 30, 1345 (1989).

[23] J. M. Pons and J. A. Garcia, Int. J. Mod. Phys. A 15 (2000) 4681 hep-th/9908151.

[24] L. D. Faddeev, Sov. Phys. Usp. 25, 130 (1982).

[25] T. Regge and C. Teitelboim, Annals of Physics 88, 286 (1974) 